\title{
EDUCACIÓN FÍSICA Y DIVERSIDAD: UNA CUESTIÓN DE GÉNERO
}

\section{Physical Education and Diversity: A Gender Issue}

(i) María Ángeles Fernández-Villarino*1 marianfv@uvigo.es

iD Ariadna Hernaiz-Sánchez ${ }^{1}$ arihernaiz@icloud.com

\section{Eloy Villaverde-Caramés ${ }^{2}$}

eloy.villaverde@gmail.com

* Autor por correspondencia

${ }^{1}$ Universidad de Vigo, España

${ }^{2}$ Universidade da Coruña, España

Fecha recepción: 15/7/2019

Fecha aprobación: 6/11/2019

\section{Resumen}

Varias investigaciones y diferentes instituciones internacionales reconocen el papel fundamental que la Educación Física puede desarrollar en la promoción de un estilo de vida activo y saludable, así como el favorecer una actitud positiva hacia la práctica de actividad físico-deportiva. La investigación evidencia que el índice de sedentarismo en la población infantil y adolescente es elevado y preocupante, cuestiones que se acentúan más en el caso de las niñas y de las adolescentes. Si una Educación Física de calidad debe responder de manera específica a las necesidades de todas y cada una de las personas que la reciben, teniendo en cuenta las evidencias anteriores, se hace necesario un análisis en torno al género, la Educación Física y su influencia en esta parte de la población, dado que parece no tener ni el mismo significado ni la misma influencia que en el caso de los niños y de los adolescentes.

Palabras clave: actividad física, Educación Física, diversidad, género.

\section{Abstract}

Several researches and different international institutions recognize the fundamental role that Physical Education can play in promoting an active and healthy lifestyle, as well as fostering a positive attitude towards the practice of physical-sports activity. Research shows that the rate of sedentarism in the child and adolescent population is high and worrisome, issues that are more accentuated in the case of girls and female adolescents. If a quality physical education is to respond specifically to the needs of each and every person who receives it, taking into account the above evidence, an analysis of gender, physical education and its influence is needed on this part of the population, given that it seems to have neither the same meaning nor the same influence as in the case of boys and male adolescents.

Keywords: diversity, gender, physical activity, Physical Education. 


\section{FERNÁNDEZ-VILLARINO • HERNAIZ-SÁNCHEZ • VILLAVERDE-CARAMÉS}

Educación física y diversidad: una cuestión de género

\section{Introducción}

A pesar de la evidencia científica que señala los numerosos beneficios que la práctica de actividad física (AF) tiene para el desarrollo de un estilo de vida activo y saludable (OMS, 2010; Hallal et al., 2012), son muchos los adolescentes que no cumplen con las recomendaciones mínimas de práctica de AF para el desarrollo de dicho estilo de vida (OMS, 2012). Del mismo modo, se destaca que las niñas practican menos que los niños (Flintoff \& Scraton, 2006; Moreno-Murcia et al., 2012; Sterdt et al., 2013), lo que supone una problemática particular: si el índice de práctica de las niñas es bajo, probablemente sus hábitos saludables en torno a la práctica de $\mathrm{AF}$ en edades adultas sean escasos (Ackerman et al., 2018).

El género como constructo social puede influir en el disfrute de una buena salud física, mental y de bienestar, así como en el acceso a los servicios de salud y los resultados que experimentan a lo largo de la vida. Por ello se debe poner énfasis desde la investigación en evidenciar las diferencias que por esta variable se generan, pues probablemente los planes de acción e intervención deban realizarse desde una perspectiva específica, en este caso, de género. Por ejemplo, en el 2012, la OMS destacaba que la proporción de chicas de entre 11 y 15 años que no cumplía con las recomendaciones de 60 minutos de AF de moderada a vigorosa estaba entre el 81 y el 90\%. Posteriormente, en Espańa, estudios como el de Instituto Nacional de Estadística [INE] (2014), Janssen y LeBlanc (2010) y Mielgo-Ayuso et al. (2016) señalan que se continúa con esta tendencia. Estas evidencias refuerzan la idea de que el género discrimina la cultura de la motricidad, generando deficiencias en las niñas y alejándolas de la salud y el bienestar futuro (González Palomares et al., 2010).

Dentro de las diferentes estrategias que se han desarrollado para enfrentar esta situación, la World Health Organization (2008) busca en la escuela un agente transmisor en la promoción de la AF, instando par- ticularmente a las clases de EF como fuente para el desarrollo de la educación para la salud. De manera específica, entiende que un aumento en el número de clases de EF incrementaría la AF diaria de las estudiantes, así como el desarrollo de conocimientos y habilidades para la toma de decisiones que garanticen la participación a largo plazo en la AF. Todo ello en el contexto de una EF agradable, que aliente a la máxima participación de las estudiantes y donde el profesorado y todo el personal educativo tomen conciencia de que la escuela y la EF pueden ser un factor clave en la salud del futuro.

Si la escuela en general y la EF en particular generan experiencias positivas, es probable que la predisposición de las adolescentes a la práctica de AF sea mayor. Por ello, los objetivos de este estudio son: (1) describir la opinión que un grupo de adolescentes tiene frente a la escuela y la EF; (2) valorar las funciones que le otorgan a la EF escolar en función de su índice de práctica.

\section{Método}

El método utilizado para la realización de este estudio ha sido el descriptivo tipo encuesta. Esta opción metodológica responde a la necesidad de conjugar los intereses y posibilidades de quienes realizan la investigación y las exigencias y naturaleza del problema investigado (Arnal et al., 1994). Este tipo de método es de carácter fácil y directo, lo que hace que sea muy utilizado en el ámbito educativo (Cohen \& Manion, 1990).

\subsection{Participantes}

Participaron en el estudio 873 alumnas de edades comprendidas entre los 13 y los 18 años $(M=14.2$ años \pm 1.0$)$. Todas las alumnas pertenecen a centros públicos de Enseñanza Secundaria de la comunidad autónoma de Galicia, en España. La distribución por cursos se encuentra en la Tabla 1. 
Tabla 1. Distribución de la muestra por curso

\begin{tabular}{lcc}
\hline & \multicolumn{2}{c}{$N=837$} \\
& $n$ & $\%$ \\
\hline $1^{\circ}$ Secundaria & 294 & 33.7 \\
$2^{\circ}$ Secundaria & 287 & 32.9 \\
$3^{\circ}$ Secundaria & 292 & 33.4 \\
\hline
\end{tabular}

Nota: Elaboración propia.

\subsection{Variables de estudio}

Las variables estudiadas han sido: (1) Índice de práctica de AF, calculado a partir de la frecuencia y el tiempo de práctica de AF fuera de la escuelas con supervisión de entrenador/monitor o entrenadora/ monitora, la frecuencia y el tiempo de práctica de AF fuera de la escuelas con carácter recreativo y sin organizar, y la frecuencia y tiempo de práctica de AF realizada fuera del horario escolar, pero en el propio centro educativo bajo supervisión; (2) Valoración de la escuela (no me gusta nada, no me gusta mucho, me es indiferente, me gusta, me gusta mucho); (3) Valoración de la EF (no me gusta nada, no me gusta mucho, me es indiferente, me gusta, me gusta mucho); funcionalidad que le otorgan a la EF (libera energías, promueve aprendizajes o perfeccionamiento de las técnicas deportivas, enseña a tener una buena conducta moral, promueve estilos de vida activos y saludables, proporciona momentos de relajación y diversión, mejora la condición física).

\subsection{Instrumento de recolección de datos}

Como instrumento de recolección de datos se ha utilizado el CIEVA (Cuestionario Internacional sobre el Estilo de Vida de los Alumnos). Este instrumento ha sido utilizado en diferentes estudios en contextos portugueses (Carreiro da Costa \& Marques, 2011; Marques \& Carreiro da Costa, 2013); españoles (Cantero, 2018; Fernández-Villarino et al., 2017; Mayor, 2018; Mourelle, 2014; Sierra et al., 2019) y ambos (Marques et al., 2017; Lago-Ballesteros et al., 2019).

El cuestionario está constituido por 39 preguntas cerradas, estructuradas en cuatro dimensiones con la siguiente distribución: datos personales (6 ítems), hábitos de vida (12 ítems), actitudes y percepciones (12 ítems) y la valoración de la escuela, la EF y la práctica de AF (9 ítems). Es en esta última dimensión en la que se ha centrado el análisis.

Como índice de consistencia interna de las preguntas del cuestionario se utilizó el coeficiente de Alpha de Cronbach, obteniendo valores de $\alpha=.78$ para todo el cuestionario y de $\alpha>.71$ en cada una de las dimensiones estudiadas.

\subsection{Procedimiento}

Este trabajo se realizó en consonancia con los patrones éticos de las Ciencias del Deporte (Harriss \& Atkinson, 2015) y fue aprobado por la Secretaría Xeral para el Deporte de la Xunta de Galicia, institución del gobierno regional responsable de la práctica de actividad físico-deportiva en los centros escolares. También recibió la aprobación de la Universidade da Coruña, al estar bajo el marco de realización de una propuesta general en el seno de la Red Euroamericana de Actividad Física, Educación y Salud (REAFES).

Para la recolección de datos, se estableció contacto telefónico con las direcciones de los centros participantes y se les envió documentación relativa al objeto de estudio y procedimiento. Del mismo modo, también se les garantizó el anonimato, tanto de los centros participantes como del alumnado.

Una vez autorizados por la dirección del centro, se obtuvieron los consentimientos informados de los progenitores o responsables legales del alumnado participante. El cuestionario se aplicó a grupos aula de manera independiente, bajo la coordinación de la dirección del centro educativo y con la presencia del profesorado de EF.

\subsection{Análisis estadístico}

Se realizó un análisis descriptivo (frecuencias, porcentajes, medias y desviaciones típicas en función de la naturaleza de las variables). Con la intención de identificar las diferencias entre los grupos analizados se utilizó la prueba de Chi-cuadrado.

Los análisis estadísticos realizados se llevaron a cabo utilizando el programa SPSS para Windows, en su 


\section{FERNÁNDEZ-VILLARINO • HERNAIZ-SÁNCHEZ • VILLAVERDE-CARAMÉS}

Educación física y diversidad: una cuestión de género

versión 20.2 (SPSS INC. IBM, USA), con unos niveles de significatividad de $p<.05$.

\section{Resultados}

En la Tabla 2 se han incluido las variables que nos permiten calcular el índice de AF de las personas participantes en el estudio. En relación con la frecuencia de práctica de AF, fuera del centro escolar sin entrenador, más de la mitad de las encuestadas (57.7\%) afirma que practica una vez por semana, o de dos a tres veces por semana. Además, el $87.9 \%$ afirma que el máximo de tiempo que emplea en la práctica es de dos a tres horas en toda la semana.
Las chicas que nunca practican fuera del centro escolar sin entrenador suponen el 50\%. En el $71.3 \%$ de los casos no superan las tres horas por semana.

Más del 70\% de las encuestadas afirma que nunca practica AF en el centro, pero fuera del horario escolar el tiempo que invierte la mayoría es inferior a tres horas por semana.

Teniendo en cuenta estos resultados, y tomando como referencia las recomendaciones de la OMS (2010) para estas edades (60 minutos de AF diaria de moderada a vigorosa), el $76.4 \%$ de las chicas son sedentarias, frente al $23.6 \%$ que son activas.

\section{Tabla 2. Descripción de la práctica de actividad física y deportiva en diferentes contextos}

\begin{tabular}{|c|c|c|c|}
\hline & \multirow{2}{*}{$N$} & \multicolumn{2}{|c|}{ Chicas } \\
\hline & & \multicolumn{2}{|l|}{$\%$} \\
\hline \multirow{6}{*}{$\begin{array}{c}\text { Frecuencia de AF fuera del } \\
\text { centro escolar sin super- } \\
\text { visión }\end{array}$} & Nunca & 66 & 7.8 \\
\hline & -1 vez/semana & 73 & 8.6 \\
\hline & $1 \mathrm{vez} / \mathrm{semana}$ & 224 & 26.5 \\
\hline & $2 / 3$ veces/semana & 264 & 31.2 \\
\hline & $4 / 6$ veces/semana & 120 & 14.2 \\
\hline & Todos los días & 98 & 11.6 \\
\hline \multirow{5}{*}{$\begin{array}{c}\text { Tiempo de AF fuera del } \\
\text { centro escolar sin super- } \\
\text { visión }\end{array}$} & $1 / 2$ hora & 155 & 19.7 \\
\hline & 1 hora & 292 & 37.2 \\
\hline & $2-3$ horas & 244 & 31.0 \\
\hline & 4-6 horas & 67 & 8.5 \\
\hline & 7 horas o más & 28 & 3.6 \\
\hline \multirow{6}{*}{$\begin{array}{c}\text { Frecuencia de AF fuera del } \\
\text { centro escolar con super- } \\
\text { visión }\end{array}$} & Nunca & 418 & 49.9 \\
\hline & -1 vez/semana & 15 & 1.8 \\
\hline & $1 \mathrm{vez} / \mathrm{semana}$ & 99 & 11.8 \\
\hline & $2 / 3$ veces/semana & 156 & 18.6 \\
\hline & $4 / 6$ veces/semana & 121 & 14.4 \\
\hline & Todos los días & 29 & 3.5 \\
\hline \multirow{5}{*}{$\begin{array}{c}\text { Tiempo de AF fuera del } \\
\text { centro escolar con super- } \\
\text { visión }\end{array}$} & $1 / 2$ hora & 24 & 5.6 \\
\hline & 1 hora & 103 & 23.8 \\
\hline & $2-3$ horas & 181 & 41.9 \\
\hline & 4-6 horas & 67 & 15.5 \\
\hline & 7 horas o más & 57 & 13.2 \\
\hline
\end{tabular}


Revista Caribeña de Investigación Educativa | 2019, 3(2), 46-55

\begin{tabular}{|c|c|c|c|}
\hline \multirow{3}{*}{$N$} & \multirow{3}{*}{ V } & & \\
\hline & & \multicolumn{2}{|c|}{ Chicas } \\
\hline & & \multicolumn{2}{|l|}{$\%$} \\
\hline \multirow{6}{*}{$\begin{array}{l}\text { Frecuencia de AF dentro } \\
\text { del centro escolar }\end{array}$} & Nunca & 564 & 71.4 \\
\hline & -1 vez/semana & 20 & 2.5 \\
\hline & $1 \mathrm{vez} / \mathrm{semana}$ & 35 & 4.4 \\
\hline & $2 / 3$ veces/semana & 122 & 15.4 \\
\hline & $4 / 6$ veces/semana & 37 & 4.7 \\
\hline & Todos los días & 12 & 1.5 \\
\hline \multirow{5}{*}{$\begin{array}{l}\text { Tiempo de AF dentro del } \\
\text { centro escolar }\end{array}$} & $1 / 2$ hora & 58 & 25.9 \\
\hline & 1 hora & 76 & 33.9 \\
\hline & 2-3 horas & 70 & 31.3 \\
\hline & 4-6 horas & 14 & 6.3 \\
\hline & 7 horas o más & 6 & 2.7 \\
\hline
\end{tabular}

Nota: Elaboración propia.

La opinión sobre la escuela se describe en la Tabla 3. Algo más de la mitad de las encuestadas $(55.9 \%$ de las sedentarias y el $54.2 \%$ de las activas) señala que la escuela o no les gusta o les es indiferente, frente al resto de las alumnas que afirma que al menos la escuela les gusta mucho. No se han encontrado diferencias estadísticamente significativas $(p<.05)$ entre las activas y las sedentarias.

\section{Tabla 3. Opinión de las alumnas en torno a la escuela}

\begin{tabular}{|c|c|c|c|c|c|c|}
\hline & \multirow{2}{*}{\multicolumn{2}{|c|}{ Sedentarias }} & & & \multirow[b]{3}{*}{$x^{2}$} & \multirow[b]{3}{*}{$P$} \\
\hline & & & \multicolumn{2}{|c|}{ Activas } & & \\
\hline & $n$ & $\%$ & $n$ & $\%$ & & \\
\hline No me gusta nada & 50 & 7.9 & 9 & 7.6 & & \\
\hline No me gusta mucho & 101 & 15.9 & 22 & 18.6 & & \\
\hline Me es indiferente & 204 & 32.1 & 33 & 28.0 & & \\
\hline Me gusta & 233 & 36.7 & 42 & 35.6 & 2.018 & .733 \\
\hline Me gusta mucho & 47 & 7.4 & 12 & 10.2 & & \\
\hline
\end{tabular}

Nota: Elaboración propia.

En la Tabla 4 se describe la opinión sobre las clases de EF. No se han encontrado diferencias estadísticamente significativas $(p<.05)$ en torno a esta valoración y el índice de AF de las adolescentes. Cerca del
$80 \%$ de los casos $(76.9 \%$ de las sedentarias y $79.7 \%$ de las activas) les gustan o les gustan mucho las clases de EF; frente a ellas, el $8 \%$ afirma que no les gusta mucho o nada $(8.2 \%$ de las sedentarias y $8.3 \%$ de las activas). 


\section{FERNÁNDEZ-VILLARINO • HERNAIZ-SÁNCHEZ • VILLAVERDE-CARAMÉS}

Educación física y diversidad: una cuestión de género

Tabla 4. Opinión sobre las clases de Educación Física

\begin{tabular}{lccccccc}
\cline { 2 - 5 } & \multicolumn{9}{c}{ Sedentarias } & \multicolumn{3}{c}{ Activas } & & \\
\cline { 2 - 5 } & $n$ & $\%$ & $n$ & $\%$ & $X^{2}$ & $P$ \\
\hline No me gusta nada & 19 & 3.0 & 3 & 2.5 & & \\
No me gusta mucho & 33 & 5.2 & 7 & 5.9 & & \\
Me es indiferente & 95 & 14.9 & 14 & 11.9 & & \\
Me gusta & 265 & 41.7 & 54 & 45.8 & 1.266 & .867 \\
Me gusta mucho & 224 & 35.2 & 40 & 33.9 & & \\
\hline
\end{tabular}

Nota: Elaboración propia.

La funcionalidad que las alumnas encuestadas le encuentran a la EF escolar se describe en la Tabla 5. Para el 35.2\% de las alumnas sedentarias y el 30.3\% de las activas, la EF escolar sirve para promover un estilo de vida saludable. En el caso de las sedentarias, destacan la mejora de la condición física (20.4\%) y el aprendizaje y perfeccionamiento de técnicas de- portivas (16.2\%). Las funcionalidades que menos valoran las alumnas activas son el aprendizaje de una buena conducta moral y el proporcionar momentos de diversión y relajación ( $4.4 \%$ en ambos casos). No se han encontrado diferencias significativas entre activas y sedentarias $(p<.05)$.

\section{Tabla 5. Funcionalidad de la Educación Física}

\begin{tabular}{lccccccc} 
& \multicolumn{3}{c}{ Sedentarias } & \multicolumn{2}{c}{ Activas } & & \\
\cline { 2 - 5 } & $n$ & $\%$ & $n$ & $\%$ & & $X^{2}$ & \\
\hline Liberar energías & 50 & 10.0 & 12 & 13.3 & & \\
Aprendizaje técnicas deportivas & 80 & 16.2 & 17 & 18.8 & & \\
Buena conducta moral & 13 & 2.6 & 4 & 4.4 & & \\
Promoción estilos de vida saludables & 176 & 35.2 & 27 & 30.3 & & \\
Relajación y diversión & 34 & 6.8 & 4 & 4.4 & 3.896 & \\
Mejora de la condición física & 102 & 20.4 & 15 & 16.6 & & \\
No tengo opinión & 44 & 8.8 & 11 & 12.2 & & \\
\hline
\end{tabular}

Nota: Elaboración propia.

\section{Discusión y conclusiones}

Los objetivos de este estudio fueron describir la opinión de un grupo de chicas adolescentes en relación con la EF y la escuela, así como valorar la funcionalidad que le otorgan a la EF en función de su índice de práctica. El conocimiento de estas opiniones permitirá identificar el posicionamiento del grupo de alumnas de cara a entender sus actitudes ante la AF, y saber si la opinión que tienen está en línea con las recomendaciones para una EF que respeta la diversidad, concretamente, la de género.
En general, los resultados sugieren que las alumnas que son activas no llegan al 30\%. Estos resultados parecen estar en línea con los presentados por Lima-Serrano et al. (2015), en los cuales sólo el $21.4 \%$ del alumnado realizaba práctica diaria de AF de moderada a vigorosa. Estos datos contribuyen a evidenciar que el índice de práctica de AF de las niñas es bajo. Varios han sido los planes que desde diferentes instituciones se han puesto en marcha para paliar esta situación, sin embargo, parece que no están teniendo la suficiente eficacia. 
La práctica mayoritaria de actividad físico-deportiva por parte de las alumnas se realiza fuera del centro escolar, con una frecuencia no superior a las tres veces por semana y una duración total por debajo de las tres horas. Estos datos concuerdan con los presentados por el estudio ELOIN (Ortiz-Marrón et al., 2016), en el que el número de horas de práctica de AF está en el orden de las 2.7. Una vez más se pone de manifiesto la baja frecuencia y la escasa duración de la práctica de actividad físico-deportiva en las chicas.

Parece que las actividades deportivas ofertadas en los centros escolares no cumplen las expectativas de las alumnas, ya que el número que afirma realizar práctica en el centro escolar fuera del horario es escaso. Trabajos como los de Jose et al. (2011) y el Estudio Aladino (2016) señalan que a las niñas que no les gusta practicar deportes en la escuela tienden a ser menos activas en la edad adulta. Por ello se considera que la escuela puede influir en la percepción de la actitud para los deportes (Telama et el., 2014), de ahí la necesidad de revisar la oferta de práctica de actividades físico-deportivas fuera del horario escolar.

La opinión que las alumnas presentan sobre la escuela es neutra respecto a la que presentan sobre la EF. Mientras que la mayoría opina que la escuela no les gusta mucho o les es indiferente, las clases de EF son valoradas positivamente por la mayoría de las alumnas encuestadas. Según Cleland et al. (2012), un incremento en el horario de las clases de EF podría llevar a un incremento de hasta el 50\% en las actividad físico-deportiva en la edad adulta. Del mismo modo, la World Health Organization (2008) recomienda que en las clases de EF se responda a las necesidades e intereses de la edad, género y nivel de habilidad, buscando la máxima participación de las estudiantes.

A la hora de seleccionar las funcionalidades que tiene la EF, las alumnas han identificado la promoción de un estilo de vida saludable como la principal. En esta línea, la World Health Organization (2008, 2018) considera que la EF contribuye con los niveles de actividad física recomendados para el desarrollo de un estilo de vida activo y saludable. La escuela debería establecer estándares que garanticen la cali- dad de la EF para poder asegurar el potencial de esta materia como promotora de la actividad física entre el alumnado. Otra institución internacional como la UNESCO (2015) habla de experiencias de aprendizaje inclusivo dentro del aula de EF, fomentando actitudes positivas que puedan conducir a una vida más placentera y físicamente activa. Es por ello que se debe hacer una mención especial a las cuestiones de género. Para asegurar una EF de calidad se darán oportunidades al alumnado que de manera particular más lo necesite. Este es el caso de las chicas, que, como se ha reflejado en la investigación, presentan índices muy bajos de actividad física.

Por otro lado, se debe considerar que la problemática descrita no es exclusiva de la EF, de ahí que las soluciones no pueden venir únicamente desde esta perspectiva. Es en la educación para la salud y en una visión global de la problemática donde se deben buscar soluciones. Una intervención integral, con ejes sociales, sanitarios y educativos, debería fomentar el desarrollo de oportunidades de aprendizaje dirigidas a una alfabetización sanitaria. Así se podría mejorar el conocimiento, la autonomía y el desarrollo de habilidades que generarían en la población una mejora de la salud. Del mismo modo, disponer de recursos adecuados, formar a los profesionales responsables y generar una cultura de promoción de la salud se hace determinante para el éxito.

La importancia de realizar un análisis específico de la problemática de las chicas radica en que podemos focalizar la investigación. Así se dan más oportunidades de comprender una realidad que se estudia desde hace tiempo y que no se consigue transformar a pesar de los intentos. Parece que la EF ha dirigido parte de sus esfuerzos a responder a las necesidades de este sector poblacional y está consiguiendo romper con los estereotipos, o con la prevalencia/reproducción de los estereotipos, en torno a la práctica de actividad físico-deportiva en el caso de las niñas (Alvariñas Villaverde \& Novoa, 2016).

Sería interesante disponer de indicadores específicos que nos permitan interpretar la realidad de las chicas 


\section{FERNÁNDEZ-VILLARINO • HERNAIZ-SÁNCHEZ • VILLAVERDE-CARAMÉS}

Educación física y diversidad: una cuestión de género

que no hacen AF suficiente. De este modo despertaríamos la sensibilidad que nos permita avanzar en soluciones, generando un conocimiento más específico del problema.

\section{Referencias}

Ackerman, K. E., Holtzman, B., \& Parziale, A. L. (2018). Actividad física en la infancia y la adolescencia: beneficios inmediatos y a largo plazo. En E. Ferrer (Ed.). Salud y Deporte en Femenino. La importancia de mantenerse activa desde la infancia. Hospital San Joan de Déu. http://bit.ly/2qF66gI

Agencia Española de Consumo, Seguridad Alimentaria y Nutrición. (2016). Estudio Aladino 2015: Estudio de vigilancia del crecimiento, alimentación, actividad física, desarrollo infantil y obesidad en España 2015. Ministerio de Sanidad, Servicios Sociales e Igualdad. https://bit.ly/2Rjoqqw

Alvariñas Villaverde, M., \& Novoa Pérez, A. (2016). Pensamientos relacionados con la actividad física y el género en adolescentes de Galicia. Sportis. Scientific Journal of School Sport, Physical Education and Psychomotricity, 2(1), 23-35.

https://doi.org/10.17979/sportis.2016.2.1.1439

Arnal, J., del Rincón, D., \& Latorre, A. (1994). Investigación educativa: Fundamentos y Metodología. Labor.

Cantero Castrillo, P. (2018). La percepción de salud en jóvenes escolares (Tesis doctoral). Universidade da Coruña, A Coruña. https://bit.ly/363oKOh

Cleland, V., Dwyer, T., \& Venn, A. (2012). Which domains of childhood physical activity predict physical activity in adulthood? A 20-year prospective tracking study. British Journal of Sports Medicine, 46(8), 595-602.

https://doi.org/10.1136/bjsports-2011-090508

Cohen, L., \& Manion, L. (1990). Métodos de investigación educativa. Editorial La Muralla.

Carreiro da Costa, F., \& Marques, A. (2011). Promoting active and healthy lifestyle at school: views of students, teachers, and parents in Portugal. In K.
Hardman \& K. Green (Eds.). Contemporary Issues in Physical Education: International Perspectives (pp. 249-268). Meyer \& Meyer Sport.

https://bit.ly/33QREzw

Fernández-Villarino, M. Á., González Valeiro, M., Toja Reboredo, B., \& Carreiro da Costa, F. (2017). Valoración de la escuela y la Educación Física y su relación con la práctica de actividad física de los escolares. Retos: Nuevas Tendencias en Educación Física, Deporte y Recreación, (31), 312-315.

https://bit.ly/2Li5Fjx

Flintoff, A., \& Scraton, S. (2006). Girls and physical education. In D. Kirk, D. Macdonald \& M. O'Sullivan (Eds.). The handbook of physical education (pp. 767-783). SAGE.

https://doi.org/10.4135/9781848608009.n43

González Palomares, A., Táboas Pais, M. I., Rey Cao, A. (2010). Los libros de texto como herramientas para la promoción de una práctica físico-deportiva en igualdad: análisis comparativo de la representación racial entre los libros publicados durante la vigencia de la LOGSE y la LOE. Cuadernos de Psicología del Deporte, 10(2). https://bit.ly/2DXhZ4N

Hallal, P. C., Andersen, L. B., Bull, F. C., Guthold, R., Haskell, W., \& Ekelund, U. (2012). Global physical activity levels: surveillance progress, pitfalls, and prospects. The Lancet, 380(9838), 247-257. https://doi.org/10.1016/S0140-6736(12)60646-1

Harriss, D. J., \& Atkinson, G. (2015). Ethical standards in sport and exercise science research: 2016 update. International Journal of Sports Medicine, 36(14), 1121-1124.

https://doi.org/10.1055/s-0035-1565186ç

Instituto Nacional de Estadística [INE] (2014). Encuesta Europea de Salud en España 2014. Ministerio de Sanidad Consumo y Bienestar social.

https://bit.ly/38CPUOc

Janssen, I., \& LeBlanc, A. G. (2010). Systematic review of the health benefits of physical activity and fitness in school-aged children and youth. International journal of Behavioral Nutrition and Physical 


\section{Revista Caribeña de Investigación Educativa | 2019, 3(2), 46-55}

Activity, 7(40).

https://doi.org/10.1186/1479-5868-7-40

Jose, K. A., Blizzard, L., Dwyer, T., McKercher, Ch., \& Venn, A. J. (2011). Childhood and adolescent predictors of leisure time physical activity during the transition from adolescence to adulthood: a population based cohort study. International Journal of Behavioral Nutrition and Physical Activity, 8(54). https://doi.org/10.1186/1479-5868-8-54

Lago-Ballesteros, J., Martins, J., González-Valeiro, M. Á., \& Fernández-Villarino, M. A. (2019). Parental assessment of physical education in the school curriculum: A brief report on the influence of past experiences as students. PloS ONE, 14(7). https://doi.org/10.1371/journal.pone.0219544

Lima-Serrano, M., Guerra-Martín, M. D., \& Lima-Rodríguez, J. S. (2015). Estilos de vida y factores asociados a la alimentación y la actividad física en adolescentes. Nutrición Hospitalaria, 32(6), 2838-2847. http://dx.doi.org/10.3305/nh.2015.32.6.9831

Marques, A., \& Carreiro da Costa, F. (2013). Levels of physical activity of urban adolescents according to age and gender. International Journal of Sports Science, 3(1), 23-27. https://bit.ly/2qqgSY8

Marques, A., González Valeiro, M., Martins, J., Fernández-Villarino, M. A., \& Carreiro da Costa, F. (2017). Relación entre la actividad física de los adolescentes y la de madres/padres. Revista de Psicología del Deporte, 26(1), 145-156. https://bit.ly/2Pcss1o

Mayor Villalaín, A. (2018). Los proyectos deportivos como medio de promoción de actividad física en los centros escolares gallegos: tratamiento desde una perspectiva de género. (Tesis doctoral). Universidade da Coruña, A Coruña. https://bit.ly/2YrbnFf

Mielgo-Ayuso, J., Aparicio-Ugarriza, R., Castillo, A., Ruiz, E., Ávila, J. M., Aranceta-Batrina, J., Gil, A., Ortega, R., Serra-Majem, L., Varela-Moreiras, G., \& González-Gross, M. (2016). Physical activity patterns of the Spanish population are mostly determined by sex and age: findings in the ANIBES study. PLoS ONE, 11(2). https://doi.org/10.1371/journal.pone.0149969

Moreno-Murcia, J. A., Huéscar, E., \& Cervelló, E. (2012). Prediction of adolescents doing physical activity after completing secondary education. The Spanish Journal of Psychology, 15(1), 90-100.

https://doi.org/10.5209/rev_sjop.2012.v15.n1.37288

Mourelle, M. (2014). Relación de los agentes sociales con el indice de práctica de actividad física de los escolares de 10 a 16 años de las urbes gallegas (Tesis doctoral). Universidade da Coruña, A Coruña. https://bit.ly/2Lix6d1

Organización Mundial de la Salud (2010). Recomendaciones mundiales sobre actividad física para la salud. Organización Mundial de la Salud. http://bit.ly/2E6mKsz

Organización Mundial de la Salud (2012). Social determinants of health and well-being among young people. Health behavior in school-aged children (HBSC) study: International report from the 2009/2010 survey. Organización Mundial de la Salud. http://bit.ly/2qIxXN5

Ortiz-Marrón, H., Cuadrado-Gamarra, J. I., Esteban-Vasallo, M., Cortés-Rico, O., Sánchez-Díaz, J., \& Galán-Labaca, I. (2016). Estudio Longitudinal de Obesidad Infantil (ELOIN): diseño, participación y características de la muestra. Revista Española de Cardiología, 69(5), 521-523.

https://doi.org/10.1016/j.recesp.2016.01.018

Sierra Palmeiro, E., González Valeiro, M. A., \& Fernández-Villarino, M. (2019). Overweight in schoolchildren and association with physical activity and parental habits. Revista Brasileira de Medicina do Esporte, 25(4), 290-294.

https://doi.org/10.1590/1517-869220192504 181165

Sterdt, E., Liersch, S., \& Walter, U. (2013). Correlates of physical activity of children and adolescents: a systematic review of reviews. Health Education Journal, 73(1), 72-89.

https://doi.org/10.1177/0017896912469578

Telama, R., Yang, X., Leskinen, E., Kankaanpää, A., Hirvensalo, M., Tammelin, T., Viikari, J., \& Rai- 


\section{FERNÁNDEZ-VILLARINO • HERNAIZ-SÁNCHEZ • VILLAVERDE-CARAMÉS}

Educación física y diversidad: una cuestión de género

takari, O. (2014). Tracking of physical activity from early childhood through youth into adulthood. $M e-$ dicine \& Science in Sports \& Exercise, 46(5), 955-962. https://doi.org/10.1249/mss.0000000000000181

UNESCO (2015). Quality physical education (QPE). Guidelines for policy-makers. United Nations Educational, Scientific and Cultural Organization. https://bit.ly/2qkSiaV
World Health Organization (2008). School policy framework: Implementation of the WHO global strategy on diet, physical activity and health. WHO Press, World Health Organization. https://bit.ly/2PiwpBs

World Health Organization (2018). Promoting physical activity in the education sector. WHO regional Office for Europe. https://bit.ly/33NDC1J

\section{CÓMO CITAR:}

Fernández-Villarino, M. A., Hernaiz-Sánchez, A., \& Villaverde-Caramés, E. (2019). Educación Física y Diversidad: una cuestión de género. Revista Caribeña de Investigación Educativa (RECIE), 3(2), 46-55. https://doi.org/10.32541/recie.2019. v3i2.pp46-55 